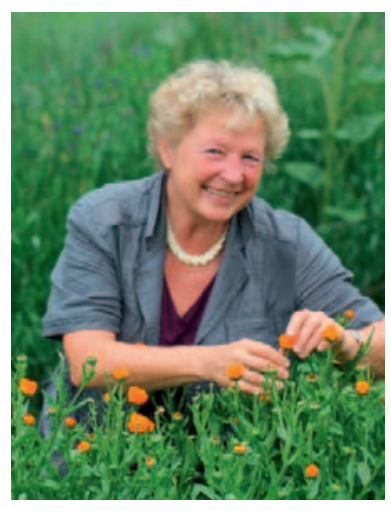

Ursel Bühring,

Freiburg i.Br., Deutschland

\section{Gesund bleiben}

«Nur Gesundung ist noch schöner als Gesundheit.»

Es stimmt, was Gerhard Kocher, der schweizerische Publizist, Politologe und Gesundheitsökonom, der 1976 die Schweizerische Gesellschaft für Gesundheitspolitik gründete, sagte. Zumindest schätzt man Gesundheit nach der Gesundung noch mehr. Wobei die Gesundheit ist wie Salz: Man merkt es erst, wenn es fehlt. Gesund sein und bleiben kommt vor dem Gesunden. Nicht oder weniger schnell krank sein - und wenn, dann rasch wieder gesund werden - möchten wir alle. Was in jungen Jahren meist als Selbstverständlichkeit gilt, d.h. gesund sein, gehört mit zunehmendem Alter zur Lebensaufgabe: gesund bleiben. Zunehmend bekommt die Gesundheit einen höheren Stellenwert. Ist das ein Editorial wert?

«Wer nicht jeden Tag etwas Zeit für seine Gesundheit aufbringt, muss eines Tages sehr viel Zeit für seine Krankheit opfern», postulierte einst Sebastian Kneipp.

Es kommt wohl gerade zur rechten Zeit, dass die KneippTherapie von der Deutschen UNESCO-Kommission als «Immaterielles Kulturerbe» anerkannt wurde. Es ist also etwas wert, ein Kulturerbe gar, wenn Menschen (die Verantwortung über) ihre Gesundheit wieder selbst in die Hand nehmen - oder dazu angelernt werden! Es geht «bei Kneipp» um das Vorbeugen und Heilen anhand seiner fünf Säulen, die sich so oder ähnlich in vielen anderen Gesundheitsmodellen finden, nahezu weltweit: Neben Bewegung und Wassertherapie gehören Ernährung, Ordnungstherapie und der Einsatz von Heilpflanzen dazu. Dies alles, um die Selbstheilungskräfte, die Lebensqualität und die Res- sourcen des Menschen in seiner Gesamtheit zu unterstützen, damit Krankheiten möglichst nicht eintreten. Wie früher in China, als Ärzte nur entlohnt worden seien, wenn ihre Patienten gesund blieben. Vielleicht handelt es sich hierbei um einen Mythos, zumindest aber hatte die Medizin damals einen eher präventiven statt reparativen Ansatz.

Moderne Gesundheitsversorgung als Teil der Komplementärmedizin muss ein Interesse an der Gesundheit der Menschen haben, wobei Dienstleistungen an Kranken und an Gesunden honoriert gehören. Wenn es dabei auch um gezielte Massnahmen zum Erhalt und zur Förderung der Motivation durch geschulte Therapeuten oder «Gesundheitscoaches» geht, gehört für mich als Würze eine Prise «Lust am Tun» und wenn möglich «in Gemeinschaft» dazu. Denn wer Freude daran hat und sich mit anderen zusammentut in Sachen Bewegung, gesundes Genuss-Essen und ausgewogene Work-Life-Balance (die heutige Ordnungstherapie), der überwindet den «inneren Schweinehund» und bleibt auch dabei. So wie man in der Medizin sagt, «Eine Therapie ist nur so gut, wie sie auch an- oder eingenommen werden kann», gilt auch hier: Die Akzeptanz und damit die Compliance spielen eine grosse Rolle in der Gesundheitsvorsorge und -fürsorge. Das funktioniert nie mit moralischem Zeigefinger, dafür aber mit Lust an der Sache, denn dann ist Gesundbleiben ein Genuss. Recht hat der Philosoph Arthur Schopenhauer (1788-1860), wenn er sagt:

«Neun Zehntel unseres Glückes beruhen allein auf der Gesundheit. Mit ihr wird alles eine Quelle des Genusses. Hingegen ist ohne sie kein äusseres Gut, welcher Art auch, geniessbar.»

Wir sind heute in der Lage, die kompliziertesten Krankheiten zu behandeln - aber können wir nicht mehr dafür tun, die Menschen gesund zu erhalten? Immer wieder mag es eine Frage des «cui bono» (wem nutzt es) sein, wenn wir überlegen, wofür Gelder freigemacht, wofür Studien initiiert werden. Wer profitiert, wenn der Mensch gesund bleibt? Ohne ins Detail zu gehen, muss die Antwort stets lauten, dass zuerst einmal jeder für sich selbst verantwortlich ist.

Ein Teil der Bevölkerung verhält sich heute viel gesundheitsbewusster als früher: Man kümmert sich um seine eigene Gesundheit vor allem mittels Fitness oder auch Entspannung. Man lernt (wieder) zu kochen, selbst Kinder lernen das - als Alternative zu Fertiggerichten. Vorbeugen für die eigene Gesundheit soll sein wie kochen und danach geniessen. Ich nehme das Zepter selbst in die Hand und suche mir heraus, was mir gut tut und mir liegt, ganz individuell.

\section{KARGER}

(c) 2016 S. Karger GmbH, Freiburg

Fax +497614520714 


\section{Mein Plädoyer: Vorbeugen mit Heilpflanzen}

Heilpflanzen können Krankheiten lindern oder heilen; sie mindern Symptome und machen das Leben erträglicher oder wieder richtig lebenswert. Kostbar ist zudem: Sie dienen auch dem Wohlbefinden, sie fördern Lebensqualität, sie halten gesund, sie pflegen - und sie schmecken!

Heilpflanzen essen (statt teure und dicke Nahrungsergänzungspillen herunterzuwürgen) und regelmässig Heilpflanzenkuren durchführen, das ist meine Empfehlung. Und zwar nicht erst, wenn der Mensch erkrankt ist, sondern um beispielsweise innere Organe zu pflegen und solchermassen gesund zu erhalten.

\section{Heilpflanzen essen}

Ein feinaromatischer Bittertrunk als Lebenselixier, Smoothie oder Pesto als moderne Türöffner für wirkkräftiges «Wildkräuter-Essen», Genuss-Tees mit Arzneipflanzen (Ackerstiefmütterchen, Fenchel, Ringelblume, Rosmarin, Zitronenmelisse: alle heilkräftig und wohlschmeckend), Wildkräutergenuss in jeder Form, samt essbaren (gesunden!) Blüten - der Beispiele und Bücher gibt es heutzutage genügend.

\section{Heilpflanzenkuren}

Kuren dienen einerseits dem weitgehend gesunden Menschen als Vorsorge und andererseits demjenigen, der schon ernsthaft krank ist, zur Genesung. So bekommt der Körper einen positiven Impuls und die Seele eine Auszeit. Heilpflanzenkuren wendet man über eine begrenzte Zeit an (normalerweise 4 Wochen) und fördert so die Erhaltung der Gesundheit. Grundvoraussetzung ist, dass sie nur mit solchen Arzneipflanzen durchgeführt werden, die in der Kurzeit und in einer vernünftigen Dosierung keine Nebenwirkungen entfalten. Ein paar Beispiele: Wer sein (nicht krankes!) Herz unterstützen möchte, kurt mit Weissdorn - dieser ist bestens für die Selbstmedikation geeignet! Wer seine strapazierte (nicht kranke!) Leber gesund halten möchte, kurt mit Mariendistel, einem zugleich hocheffizienten Antioxidans und Zellschützer. Knoblauch und Buchweizenteekuren sorgen für gesunde Gefässe - denn der Mensch ist so jung wie seine Gefässe -, und zur Stärkung bei übergrosser Belastung oder Stress kann eine «Adaptogen-Kur» mit Rosenwurz prophylaktisch durchgeführt werden, um eben nicht zu erkranken (darin liegt ja die Stärke der Adaptogene). Und warum eigentlich nicht die Nieren pflegen mit einer vierwöchigen Goldruten-Kur?

Die bekannteste Kur ist die Frühjahrskur zum Start in das neue Jahr. Gärtner wissen, wie wichtig gerade in der Wachstumsphase des Frühlings guter Boden ist, um im Sommer ertragreiche Pflanzen zu haben. Sie jäten vor dem Einsäen und Bepflanzen zuerst einmal das Unkraut. Die Wurzeln einer Pflanze nehmen ihre Nährstoffe aus dem Boden auf; sind Nährstoffe und Boden gesund, wird es eine gesunde Pflanze. So ähnlich ist es mit der Frühjahrskur beim Menschen. Sind die «Wurzeln» des Menschen (Darm mitsamt Immunsystem) gesund und der Boden (unsere Nahrung) ebenso, ist der Mensch gesund. Darum werden im Frühjahr die Entgiftungsorgane unterstützt - im Körper wird sozusagen «Unkraut gejätet». Nach der «Entgiftung» starten wir mit neuer Spannkraft und Beweglichkeit, abwehrbereitem Immunsystem und spürbar neuer Lebensenergie.

Das klingt seltsam, vorbeugend zu «kuren» und die inneren Organe zu pflegen? Dass wir unser Auto regelmässig in die Inspektion schicken und pflegen, in der Wohnung Frühjahrsputz und -pflege betreiben sowie Schuhe, Kleidung, Haut und Haare pflegen, ist hingegen normal ...

Vorbeugung ist mein Zauberwort, wozu Begriffe gehören wie Salutogenese oder Gesundheitspädagogik. Stets geht es um uns, solange wir noch keine Patienten sind. Es geht um unsere eigene Zukunft und unser kostbarstes Geschenk, die Gesundheit, die wir so lange wie möglich erhalten möchten. Das kommt der Gesundheitspolitik entgegen und sollte (viel mehr) belohnt und unterstützt werden und gewürdigt. Wie gerade die UNESCO-Kommission die Kneipp-Therapie gewürdigt hat.

Und da heute die Gesundheit in den Fokus gerückt wurde, kann ich nur wünschen: Bleiben Sie gesund! 